\title{
On the Number of $\tau$-Tilting Modules over Nakayama Algebras
}

\author{
Hanpeng $\mathrm{GAO}^{\dagger}$ and Ralf SCHIFFLER ${ }^{\ddagger}$ \\ $\dagger$ Department of Mathematics, Nanjing University, Nanjing 210093, P.R. China \\ E-mail: hpgao07@163.com \\ ¥Department of Mathematics, University of Connecticut, Storrs, CT 06269-1009, USA \\ E-mail: schiffler@math.uconn.edu
}

Received March 06, 2020, in final form June 11, 2020; Published online June 18, 2020

https://doi.org/10.3842/SIGMA.2020.058

\begin{abstract}
Let $\Lambda_{n}^{r}$ be the path algebra of the linearly oriented quiver of type $\mathbb{A}$ with $n$ vertices modulo the $r$-th power of the radical, and let $\widetilde{\Lambda}_{n}^{r}$ be the path algebra of the cyclically oriented quiver of type $\widetilde{\mathbb{A}}$ with $n$ vertices modulo the $r$-th power of the radical. Adachi gave a recurrence relation for the number of $\tau$-tilting modules over $\Lambda_{n}^{r}$. In this paper, we show that the same recurrence relation also holds for the number of $\tau$-tilting modules over $\widetilde{\Lambda}_{n}^{r}$. As an application, we give a new proof for a result by Asai on recurrence formulae for the number of support $\tau$-tilting modules over $\Lambda_{n}^{r}$ and $\widetilde{\Lambda}_{n}^{r}$.
\end{abstract}

Key words: $\tau$-tilting modules; support $\tau$-tilting modules; Nakayama algebras

2020 Mathematics Subject Classification: 16G20; 16G60

\section{Introduction}

The starting point of tilting theory was the introduction of tilting modules over a hereditary algebra by Happel and Ringel in [10]. Ever since, the study of tilting modules and their endomorphism algebras has been an important branch of representation theory.

About 25 years later, cluster-tilting theory, introduced in [7] (and [8] in type $\mathbb{A}$ ), brought a new perspective to the subject by replacing the hereditary algebra by its cluster category, and a direct relation between tilted and cluster-tilted algebras was then established in [4].

In 2014, Adachi, Iyama and Reiten [2] introduced $\tau$-tilting theory replacing the rigidity condition $\operatorname{Ext}_{\Lambda}^{1}(T, T)=0$ for a tilting module by the weaker condition $\operatorname{Hom}_{\Lambda}\left(T, \tau_{\Lambda} T\right)=0$ for a $\tau$-tilting module, where $\tau$ denotes the Auslander-Reiten translation, and $\Lambda$ is any finitedimensional algebra. They showed that, in contrast to tilting modules but in agreement with cluster-tilting objects, it is always possible to exchange a given indecomposable summand of a support $\tau$-tilting module for a unique other indecomposable and obtain a new support $\tau$ tilting module. This process, called mutation, is essential in cluster theory.

In the same paper, the authors also showed that the support $\tau$-tilting modules are in bijection with several other important classes in representation theory including functorially finite torsion classes introduced in [6], 2-term silting complexes introduced in [11], and cluster-tilting objects in the cluster category when the algebra $\Lambda$ is hereditary, or, more generally, cluster-tilted.

Therefore, it is natural to ask what is the number of support $\tau$-tilting modules over a given algebra.

For a hereditary algebra, the support $\tau$-tilting modules are exactly the support tilting modules. Moreover they are in bijection with the cluster-tilting objects in the cluster category and

This paper is a contribution to the Special Issue on Cluster Algebras. The full collection is available at https://www.emis.de/journals/SIGMA/cluster-algebras.html 
hence with the clusters in the corresponding cluster algebra. For Dynkin type, these numbers were first calculated in [9] via cluster algebras, and later in [12] via representation theory. In particular, over a hereditary algebra of type $\mathbb{A}_{n}$, the number of tilting modules is $C_{n}$, and the number of support tilting modules is $C_{n+1}$ where $C_{i}$ denotes the $i$-th Catalan number $\frac{1}{i+1}\left(\begin{array}{c}2 i \\ i\end{array}\right)$.

In this paper, we study this question over finite-dimensional Nakayama algebras. Recall that a finite-dimensional $K$-algebra is said to be a Nakayama algebra if every indecomposable projective module and every indecomposable injective module has a unique composition series. Nakayama algebras come in two types, in fact, a finite-dimensional algebra is Nakayama if and only if its quiver is one of the following

$$
A_{n}: \quad 1 \longrightarrow 2 \longrightarrow 3 \rightarrow \cdots \rightarrow n, \quad \widetilde{A}_{n}: \quad 1 \longrightarrow 2 \longrightarrow 3 \rightarrow \cdots \rightarrow n,
$$

see [5, Section V.3.2]. Throughout the paper, we use the following notation

$$
\Lambda_{n}^{r}=K A_{n} / \operatorname{rad}^{r} \quad \text { and } \quad \widetilde{\Lambda}_{n}^{r}=K \widetilde{A}_{n} / \operatorname{rad}^{r} .
$$

Moreover, we let $t_{r}(n)$ and $\tilde{t}_{r}(n)$ denote the number of $\tau$-tilting modules over $\Lambda_{n}^{r}$ and $\widetilde{\Lambda}_{n}^{r}$, and let $s_{r}(n)$ and $\tilde{s}_{r}(n)$ denote the number of support $\tau$-tilting modules over $\Lambda_{n}^{r}$ and $\widetilde{\Lambda}_{n}^{r}$, respectively. We also set $t_{r}(n):=0$ and $s_{r}(n):=0$ for $n<0$.

Adachi classified $\tau$-tilting modules over Nakayama algebras in [1]. Under the assumption that the Loewy length of every indecomposable projective module is at least $n$, Adachi showed that the number of $\tau$-tilting modules is exactly $\left(\begin{array}{c}2 n-1 \\ n-1\end{array}\right)$ and the number of support $\tau$-tilting modules is $\left(\begin{array}{c}2 n \\ n\end{array}\right)$. Moreover, he also gave the following recurrence relation for the number $t_{r}(n)$ of $\tau$-tilting modules over $\Lambda_{n}^{r}$

$$
t_{r}(n)=\sum_{i=1}^{r} C_{i-1} \cdot t_{r}(n-i) .
$$

The aim of this paper is study the number of $\tau$-tilting modules over $\widetilde{\Lambda}_{n}^{r}$. We show that there is a close relationship between the number $\tilde{t}_{r}(n)$ of $\tau$-tilting $\widetilde{\Lambda}_{n}^{r}$-modules and the number $t_{r}(n)$ of $\tau$-tilting $\Lambda_{n}^{r}$-modules.

Proposition 1.1 (see Proposition 3.7).

$$
\tilde{t}_{r}(n)=\sum_{i=1}^{r} i \cdot C_{i-1} \cdot t_{r}(n-i) .
$$

Next, we prove that the functions $t$ and $\tilde{t}$ satisfy the same recurrence relation.

Theorem 1.2 (see Theorem 3.8). We have the following recurrence relation

$$
\tilde{t}_{r}(n)=\sum_{i=1}^{r} C_{i-1} \cdot \tilde{t}_{r}(n-i) .
$$

As an application, we obtain a new proof for the following result by Asai on the numbers of support $\tau$-tilting modules $s_{r}(n)$ and $\tilde{s}_{r}(n)$.

Theorem 1.3 ([3, Theorem 4.1]).

(1) $s_{r}(n)=2 s_{r}(n-1)+\sum_{i=2}^{r} C_{i-1} \cdot s_{r}(n-i)$.

(2) $\tilde{s}_{r}(n)=2 \tilde{s}_{r}(n-1)+\sum_{i=2}^{r} C_{i-1} \cdot \tilde{s}_{r}(n-i)$. 
Asai used a bijection between support $\tau$-tilting modules and semibricks to obtain his result in the context of semibricks. Our proof is combinatorial.

The paper is organized as follows. In Section 2, we fix the notation and recall several results of Adachi that are relevant to this paper. We study the Nakayama algebras of type $A_{n}$ and $\widetilde{A}_{n}$ and prove Theorem 1.2 in Section 3. Theorem 1.3 is proved in Section 4. We include tables of the numbers of $\tau$-tilting and support $\tau$-tilting modules in Section 5 .

\section{Preliminaries}

Throughout this paper, all algebras will be basic, connected, finite-dimensional algebras over an algebraically closed field $K$ and all modules will be finitely generated right modules. For an algebra $\Lambda$, we denote by $\bmod \Lambda$ the category of finitely generated right $\Lambda$-modules and by $\tau_{\Lambda}$ the Auslander-Reiten translation of $\Lambda$. Let $\left\{e_{1}, e_{2}, \ldots, e_{n}\right\}$ be a complete set of primitive orthogonal idempotents of $\Lambda$. We put $P_{i}=e_{i} \Lambda$ the indecomposable projective module and $S_{i}=P_{i} / \operatorname{rad} P_{i}$ the simple module of $\Lambda$ for $i=1,2, \ldots, n$. For $M \in \bmod \Lambda$, we denote by $\ell(M)$ the Loewy length of $M$ and by $|M|$ the number of pairwise nonisomorphic indecomposable summands of $M$. For a finite set $X$, we denote by $|X|$ the cardinality of $X$. For details on representation theory of finite-dimensional algebras we refer to $[5,13]$.

Let $\Lambda$ be an algebra. In this section, we recall results about support $\tau$-tilting modules that are needed later.

Definition 2.1. Let $M \in \bmod \Lambda$.

(1) $M$ is called $\tau$-rigid if $\operatorname{Hom}_{\Lambda}\left(M, \tau_{\Lambda} M\right)=0$.

(2) $M$ is called $\tau$-tilting if it is $\tau$-rigid and $|M|=|\Lambda|$.

(3) $M$ is called support $\tau$-tilting if it is a $\tau$-tilting $\Lambda / \Lambda e \Lambda$-module for some idempotent $e$ of $\Lambda$.

(4) $M$ is called proper support $\tau$-tilting if it is a support $\tau$-tilting but not a $\tau$-tilting $\Lambda$-module.

Recall that $M \in \bmod \Lambda$ is called sincere if every simple $\Lambda$-module appears as a composition factor in $M$. It is well-known that the $\tau$-tilting modules are exactly the sincere support $\tau$-tilting modules [2, Proposition 2.2(a)].

We will denote by $\tau$-tilt $\Lambda$ (respectively, $\operatorname{s} \tau$-tilt $\Lambda, \operatorname{ps} \tau$-tilt $\Lambda$ ) the set of isomorphism classes of basic $\tau$-tilting (respectively, support $\tau$-tilting, proper support $\tau$-tilting) $\Lambda$-modules. Obviously, we have $\mid \operatorname{s} \tau$-tilt $\Lambda|=| \tau$-tilt $\Lambda|+| \operatorname{ps} \tau$-tilt $\Lambda \mid$.

Let $\operatorname{ps} \tau$-tilt ${ }_{n p} \Lambda:=\{M \in \operatorname{ps} \tau$-tilt $\Lambda \mid M$ has no projective direct summands $\}$. We recall the following results proved by Adachi in [1].

Theorem 2.2 ([1, Theorem 2.6]). Let $\Lambda$ be a Nakayama algebra. There is a bijection between $\tau$-tilt $\Lambda$ and $\operatorname{ps} \tau$-tilt ${ }_{n p} \Lambda$.

The following result is very useful.

Proposition 2.3 ([1, Proposition 2.32]). Let $\Lambda$ be a Nakayama algebra of type $A_{n}$. Then each $\tau$-tilting $\Lambda$-module has $P_{1}$ as a direct summand.

The following recurrence relations are very useful to calculate the number of $\tau$-tilting modules over Nakayama algebras of type $A_{n}$.

Lemma 2.4 ([1, Corollary 2.34]). Let $\Lambda$ be a Nakayama algebra of type $A_{n}$. Then

$$
|\tau-\operatorname{tilt} \Lambda|=\sum_{i=1}^{\ell\left(P_{1}\right)} C_{i-1} \cdot\left|\tau-\operatorname{tilt}\left(\Lambda /\left\langle e_{\leqslant i}\right\rangle\right)\right|,
$$


where $e_{\leqslant i}:=e_{1}+e_{2}+\cdots+e_{i}$. In particular, for $\Lambda=\Lambda_{n}^{r}$, we have

$$
t_{r}(n)=\sum_{i=1}^{r} C_{i-1} \cdot t_{r}(n-i),
$$

where $t_{r}(n)$ is the number of $\tau$-tilting modules over $\Lambda_{n}^{r}$.

See Table 1 in Section 5 for explicit values of $t_{r}(n)$.

Remark 2.5. If $r \geq n$ then $\Lambda_{n}^{r}$ is hereditary, and we have $t_{r}(n)=C_{n}$. On the other hand,

Lemma 2.4 yields the equation $t_{n}(n)=\sum_{i=1}^{n} C_{i-1} \cdot t_{n}(n-i)$. Hence we recover the well-known combinatorial identity

$$
C_{n}=\sum_{i=1}^{n} C_{i-1} \cdot C_{n-i} .
$$

\section{The number of $\tau$-tilting modules over Nakayama algebras}

In this section, $\Lambda$ will be any Nakayama algebra of type $A_{n}$. As usual, we use the notation $t_{r}(n)$ for the number of $\tau$-tiling modules over $\Lambda_{n}^{r}=K A_{n} / \operatorname{rad}^{r}$ and $\widetilde{t}_{r}(n)$ for the number of $\tau$-tiling modules over $\widetilde{\Lambda}_{n}^{r}=K \widetilde{A}_{n} / \mathrm{rad}^{r}$. We will show that the functions $t$ and $\tilde{t}$ satisfy the same recurrence relation.

We denote by $W_{i}(i=1,2, \ldots, n)$ the set of support $\tau$-tilting $\Lambda$-modules which have the simples $S_{1}, S_{2}, \ldots, S_{i-1}$ as composition factor but not $S_{i}$. We also write $\Lambda_{>i}:=\Lambda /\left\langle e_{\leqslant i}\right\rangle$ and $\Lambda_{<i}:=\Lambda /\left\langle e_{\geqslant i}\right\rangle$ where $e_{\leqslant i}:=e_{1}+e_{2}+\cdots+e_{i}$ and $e_{\geqslant i}:=e_{i}+e_{i+1}+\cdots+e_{n}$.

Lemma 3.1. $\left|W_{i}\right|=\mid \tau$-tilt $\Lambda_{<i}|\cdot| \mathrm{s} \tau$-tilt $\Lambda_{>i} \mid$.

Proof. Since the quiver of $\Lambda$ is tree, we have $\Lambda /\left\langle e_{i}\right\rangle \cong \Lambda_{<i} \times \Lambda_{>i}$. Thus there is a bijection

$$
\tau \text {-tilt } \Lambda_{<i} \times \mathrm{s} \tau \text {-tilt } \Lambda_{>i} \longrightarrow W_{i}
$$

given by $\left(N_{1}, N_{2}\right) \mapsto N_{1} \oplus N_{2}$ where $N_{1}$ is a $\tau$-tilting $\Lambda_{<i}$-module and $N_{2}$ is a support $\tau$-tilting $\Lambda_{>i}$-module. Hence $\left|W_{i}\right|=\mid \tau$-tilt $\Lambda_{<i}|\cdot| \mathrm{s} \tau$-tilt $\Lambda_{>i} \mid$.

Proposition 3.2. Let $\Lambda$ be a Nakayama algebra of type $A_{n}$. We have

(1) $\mid \operatorname{ps} \tau$-tilt $\Lambda\left|=\sum_{i=1}^{n}\right| \tau$-tilt $\Lambda_{<i}|\cdot| \mathrm{s} \tau$-tilt $\Lambda_{>i} \mid$,

(2) $\mid \mathrm{s} \tau$-tilt $\Lambda\left|=\sum_{i=1}^{n}\right| \tau$-tilt $\Lambda_{<i}|\cdot| \mathrm{s} \tau$-tilt $\Lambda_{>i}\left|+\sum_{i=1}^{\ell\left(P_{1}\right)} C_{i-1} \cdot\right| \tau$-tilt $\Lambda_{>i} \mid$,

(3) $\mid \operatorname{ps} \tau$-tilt $\Lambda\left|=\sum_{i=1}^{n}\right| \mathrm{s} \tau$-tilt $\Lambda_{<i}|\cdot| \tau$-tilt $\Lambda_{>i} \mid$,

(4) $\mid \mathrm{s} \tau$-tilt $\Lambda\left|=\sum_{i=1}^{n}\right| \mathrm{s} \tau$-tilt $\Lambda_{<i}|\cdot| \tau$-tilt $\Lambda_{>i}\left|+\sum_{i=1}^{\ell\left(P_{1}\right)} C_{i-1} \cdot\right| \tau$-tilt $\Lambda_{>i} \mid$.

Proof. (1) Since $\operatorname{ps} \tau$-tilt $\Lambda=\bigcup_{i=1}^{n} W_{i}$, we have

$$
\mid \operatorname{ps} \tau \text {-tilt } \Lambda\left|=\sum_{i=1}^{n}\right| W_{i}\left|=\sum_{i=1}^{n}\right| \tau-\operatorname{tilt} \Lambda_{<i}|\cdot| \operatorname{s} \tau-\operatorname{tilt} \Lambda_{>i} \mid
$$

by Lemma 3.1.

(2) Since $\mid \operatorname{s} \tau$-tilt $\Lambda|=| \operatorname{ps} \tau$-tilt $\Lambda|+| \tau$-tilt $\Lambda \mid$, the statement follows from Lemma 2.4.

(3) is similar to (1) and (4) follows from (3). 
We give an example of Proposition 3.2.

Example 3.3. Let $\Lambda$ be an algebra is given by the quiver $1 \stackrel{\alpha}{\rightarrow} 2 \stackrel{\beta}{\rightarrow} 3 \rightarrow 4$ with the relation $\alpha \beta=0$.

\begin{tabular}{c|c|c|c|c|c|c}
\hline & $\Lambda_{<i}$ & $\Lambda_{>i}$ & $\mid \tau$-tilt $\Lambda_{<i} \mid$ & $\mid \mathrm{s} \tau$-tilt $\Lambda_{>i} \mid$ & $\mid \mathrm{s} \tau$-tilt $\Lambda_{<i} \mid$ & $\mid \tau$-tilt $\Lambda_{>i} \mid$ \\
\hline$i=1$ & 0 & $2 \stackrel{\beta}{\rightarrow} 3 \rightarrow 4$ & 1 & 14 & 1 & 5 \\
$i=2$ & 1 & $3 \rightarrow 4$ & 1 & 5 & 2 & 2 \\
$i=3$ & $1 \stackrel{\alpha}{\rightarrow} 2$ & 4 & 2 & 2 & 5 & 1 \\
$i=4$ & $1 \stackrel{\alpha}{\rightarrow} 2 \stackrel{\beta}{\rightarrow} 3, \alpha \beta=0$ & 0 & 3 & 1 & 12 & 1 \\
\hline
\end{tabular}

By Proposition 3.2(1), $\mid \operatorname{ps} \tau$-tilt $\Lambda \mid=1 \cdot 14+1 \cdot 5+2 \cdot 2+3 \cdot 1=26$. Note that $\ell\left(P_{1}\right)=2$, and thus Lemma 2.4 implies $\mid \tau$-tilt $\Lambda \mid=1 \cdot 5+1 \cdot 2=7$. Hence, we have $\mid \mathrm{s} \tau$-tilt $\Lambda \mid=26+7=33$ by Proposition 3.2(2). Moreover, we can also use part (3) of Proposition 3.2 and compute $\mid \operatorname{ps} \tau$-tilt $\Lambda \mid=1 \cdot 5+2 \cdot 2+5 \cdot 1+12 \cdot 1=26$.

Corollary 3.4. Let $V_{\ell}(\ell=1,2, \ldots, n)$ be the set of all support $\tau$-tilting $\Lambda$-modules which have $S_{\ell}, S_{\ell-1}, \ldots, S_{1}$ as composition factor. Then we have

$$
\left|V_{\ell}\right|=\sum_{i=\ell+1}^{n}\left|\tau-\operatorname{tilt} \Lambda_{<i}\right| \cdot\left|\mathrm{s} \tau-\operatorname{tilt} \Lambda_{>i}\right|+|\tau-\operatorname{tilt} \Lambda| .
$$

Proof. This result follows from $V_{\ell}=\left(\bigcup_{i=\ell+1}^{n} W_{i}\right) \bigcup \tau$-tilt $\Lambda$.

From now, we will study the number of $\tau$-tilting $\widetilde{\Lambda}_{n}^{r}$-modules. The following result is very useful to calculate the number of proper support $\tau$-tilting $\widetilde{\Lambda}_{n}^{r}$-modules.

\section{Proposition 3.5.}

$$
\left|\operatorname{ps} \tau-\operatorname{tilt} \widetilde{\Lambda}_{n}^{r}\right|=\sum_{i=1}^{n-1} i \cdot t_{r}(i-1) \cdot s_{r}(n-i-1)+n \cdot t_{r}(n-1) .
$$

Proof. For $1 \leqslant \ell \leqslant n-1$, we define

$$
K_{n, \ell}=\left\{N \in \operatorname{s} \tau-\operatorname{tilt} \widetilde{\Lambda}_{n}^{r} \mid N \text { has } S_{n}, S_{n-1}, \ldots, S_{n-\ell+1} \text { as composition factor but not } S_{n-\ell}\right\}
$$

and

$$
K_{n, 0}=\left\{N \in \operatorname{s} \tau \text {-tilt } \widetilde{\Lambda}_{n}^{r} \mid N \text { does not contain } S_{n} \text { as composition factor }\right\} .
$$

Note that $\widetilde{\Lambda}_{n}^{r} /\left\langle e_{n-\ell}\right\rangle$ is the quotient of path algebra of the quiver

$$
n-\ell+1 \longrightarrow \cdots \longrightarrow n \longrightarrow 1 \longrightarrow 2 \longrightarrow \cdots \longrightarrow n-1
$$

by the 2 -sided ideal generated by paths of length $r$. Therefore $\widetilde{\Lambda}_{n}^{r} /\left\langle e_{n-\ell}\right\rangle \cong \Lambda_{n-1}^{r}$. By Corollary 3.4 , we have

$$
\left|K_{n, \ell}\right|=\left|V_{\ell}\right|=\sum_{i=\ell+1}^{n-1} t_{r}(i-1) \cdot s_{r}(n-i-1)+t_{r}(n-1) .
$$


In particular, $\left|K_{n, 0}\right|=s_{r}(n-1)=\sum_{i=1}^{n-1} t_{r}(i-1) \cdot s_{r}(n-i-1)+t_{r}(n-1)$. Hence,

$$
\begin{aligned}
\mid \operatorname{ps} \tau \text {-tilt } \widetilde{\Lambda}_{n}^{r} \mid & =\sum_{\ell=0}^{n-1}\left|K_{n, \ell}\right|=\sum_{\ell=0}^{n-1}\left(\sum_{i=\ell+1}^{n-1} t_{r}(i-1) \cdot s_{r}(n-i-1)+t_{r}(n-1)\right) \\
& =\sum_{i=1}^{n-1} i \cdot t_{r}(i-1) \cdot s_{r}(n-i-1)+n \cdot t_{r}(n-1) .
\end{aligned}
$$

We set $X_{n}=\varnothing$, for $n<0$, and for all $n \geq 0$, we define

$$
X_{n}=\left\{N \in \mathrm{s} \tau \text {-tilt } \Lambda_{n}^{r} \mid N \text { does not contain } P_{n-r+1}, P_{n-r}, \ldots, P_{1} \text { as direct summands }\right\}
$$

and

$$
Y_{n, \ell}=\left\{N \in X_{n} \mid N \text { contains } S_{\ell}, S_{\ell-1}, \ldots, S_{1} \text { as composition factor }\right\}, \quad \ell=1,2, \ldots, n .
$$

In particular, $X_{n}=\operatorname{s} \tau$-tilt $\Lambda_{n}^{r}$ when $n+1 \leqslant r$, and $Y_{n, 0}=X_{n}$.

Lemma 3.6. With the above notions, we have

(1) $\left|X_{n}\right|=t_{r}(n+1)$,

(2) $\left|Y_{n, \ell}\right|= \begin{cases}\sum_{i=\ell+1}^{r} C_{i-1} \cdot t_{r}(n-i+1) & \text { if } \ell \leqslant r-1, \\ 0 & \text { if } \ell \geqslant r .\end{cases}$

Proof. (1) Write $\Lambda=\Lambda_{n}^{r}$, we have $\Lambda /\left\langle e_{i}\right\rangle \cong \Lambda_{<i} \times \Lambda_{>i}$ for a given $i$. Let $Z_{1}$ be the set of all support $\tau$-tilting $\Lambda_{<i}$-modules which have $S_{1}, S_{2}, \ldots, S_{i-1}$ as composition factor (they are exactly $\tau$-tilting $\Lambda_{<i}$-modules) and they don't have $P_{1}, P_{2}, \ldots, P_{n-r+1}$ as direct summands.

If $i \leqslant r$, then $Z_{1}=\tau$-tilt $\Lambda_{<i}$.

If $i \geqslant r+1$, then all $\tau$-tilting $\Lambda_{<i}$-modules have $P_{1}$ as direct summand by Proposition 2.3, and hence $Z_{1}=\varnothing$.

Let $Z_{2}$ be the set of all support $\tau$-tilting $\Lambda_{>i}$-modules who do not have $P_{1}, P_{2}, \ldots, P_{n-r+1}$ as direct summands. Then $Z_{2}$ consists of exactly all support $\tau$-tilting $\Lambda_{>i}$-modules which do not contain $P_{i+1}, P_{i+2}, \ldots, P_{n-r+1}$ as direct summands. Denoted by $X_{n, i} \subseteq X_{n}(i=1,2, \ldots, n)$ the subset of all support $\tau$-tilting modules having $S_{1}, S_{2}, \ldots, S_{i-1}$ as composition factor but not $S_{i}$ and by $X_{n, n+1} \subseteq X_{n}$ the subset of all support $\tau$-tilting modules having $S_{1}, S_{2}, \ldots, S_{n}$ as composition factor (hence, they are exactly $\tau$-tilting). We have $\left|Z_{2}\right|=\left|X_{n-i}\right|$, since $\Lambda_{>i} \cong \Lambda_{n-i}^{r}$. There is a bijection between $Z_{1} \times Z_{2}$ and $X_{n, i}$ given by $\left(N_{1}, N_{2}\right) \rightarrow N_{1} \oplus N_{2}$ where $N_{1} \in Z_{1}$ and $N_{2} \in Z_{2}$. Therefore, $\left|X_{n, i}\right|=\left|Z_{1}\right| \cdot\left|Z_{2}\right|$. Thus, we have

$$
\left|X_{n, i}\right|= \begin{cases}\left|\tau-\operatorname{tilt} \Lambda_{<i}\right| \cdot\left|X_{n-i}\right| & \text { if } i \leqslant r \\ 0 & \text { if } i \geqslant r+1 .\end{cases}
$$

If $r \leqslant n$, we have

$$
\left|X_{n}\right|=\sum_{i=1}^{n+1}\left|X_{n, i}\right|=\sum_{i=1}^{r} \mid \tau \text {-tilt } \Lambda_{<i}|\cdot| X_{n-i}\left|=\sum_{i=1}^{r} C_{i-1} \cdot\right| X_{n-i} \mid .
$$

Note that, if $r \geq n+1$ then $X_{n}=\operatorname{s} \tau$-tilt $\Lambda_{n}^{r}$ and hence $\left|X_{n}\right|=C_{n+1}$ since $\Lambda_{n}^{r}$ is hereditary, we get $\left|X_{n}\right|=t_{r}(n+1)$. On the other hand, Lemma 2.4 says that $t_{r}(n)$ and $\left|X_{n}\right|$ satisfy the same recursive formula. Thus we have $\left|X_{n}\right|=t_{r}(n+1)$ for all $n$, by induction.

(2) follows from (1) and the fact $Y_{n, \ell}=\bigcup_{i=\ell+1}^{n+1} X_{n, i}$. 
As a result of Lemma 3.6, we have the following relationship between $\tilde{t}_{r}(n)$ and $t_{r}(n)$.

Proposition 3.7. We have

$$
\tilde{t}_{r}(n)=\sum_{i=1}^{r} i \cdot C_{i-1} \cdot t_{r}(n-i) .
$$

Proof. Considering the set $K_{n, \ell}^{n p}$ consisting of all modules in $K_{n, \ell}$ which do not have projective $\widetilde{\Lambda}_{n}^{r}$-modules as direct summands.

Note that the indecomposable projective $\widetilde{\Lambda}_{n}^{r} /\left\langle e_{n-\ell}\right\rangle$-modules corresponding to the last $r-1$ points (the length of them is at most $r-1$ ) are not projective $\widetilde{\Lambda}_{n}^{r}$-modules, we have $\left|K_{n, \ell}^{n p}\right|=$ $\left|Y_{n-1, \ell}\right|$ since $\widetilde{\Lambda}_{n}^{r} /\left\langle e_{n-\ell}\right\rangle \cong \Lambda_{n-1}^{r}$. Thus,

$$
\begin{aligned}
\left|\operatorname{ps} \tau-\operatorname{tilt}_{n p} \widetilde{\Lambda}_{n}^{r}\right| & =\sum_{\ell=0}^{n-1}\left|K_{n, \ell}^{n p}\right|=\sum_{\ell=0}^{n-1}\left|Y_{n-1, \ell}\right| \\
& =\sum_{\ell=0}^{r-1} \sum_{i=l+1}^{r} C_{i-1} \cdot t_{r}(n-i) \quad(\text { by Lemma 3.6) } \\
& =\sum_{i=1}^{r} i \cdot C_{i-1} \cdot t_{r}(n-i) .
\end{aligned}
$$

Therefore, the assertion follows from Theorem 2.2.

Now, we are ready to prove our main result of this section.

Theorem 3.8. We have

$$
\tilde{t}_{r}(n)=\sum_{i=1}^{r} C_{i-1} \cdot \tilde{t}_{r}(n-i) .
$$

Proof. By Proposition 3.7, we have $\tilde{t}_{r}(n)=\sum_{\ell=1}^{r} \ell \cdot C_{\ell-1} \cdot t_{r}(n-\ell)$. Thus,

$$
\begin{aligned}
\tilde{t}_{r}(n)-\sum_{i=1}^{r} C_{i-1} \cdot \tilde{t}_{r}(n-i)= & \sum_{\ell=1}^{r} \ell \cdot C_{\ell-1} \cdot t_{r}(n-\ell) \\
& -\sum_{i=1}^{r} C_{i-1} \cdot\left(\sum_{\ell=1}^{r} \ell \cdot C_{\ell-1} \cdot t_{r}(n-i-\ell)\right) \\
= & \sum_{\ell=1}^{r} \ell \cdot C_{\ell-1} \cdot\left(t_{r}(n-\ell)-\sum_{i=1}^{r} C_{i-1} \cdot t_{r}(n-\ell-i)\right) \\
= & 0 . \quad(\text { by Lemma 2.4) }
\end{aligned}
$$

Hence, $\tilde{t}_{r}(n)=\sum_{i=1}^{r} C_{i-1} \cdot \tilde{t}_{r}(n-i)$.

The following proposition and its proof are similar to [3, Theorem 4.1 (3)]. For convenience, we include a proof here. We shall use the following notation.

For every positive integer $r$, let $F_{r}(X)=\sum_{i=0}^{r} c_{i} \cdot X^{r-i}$ where $c_{0}=1$ and $c_{i}=-C_{i-1}$ for $i=1,2, \ldots, r$. Let

$$
\mathbf{E}_{n}\left(X_{1}, X_{2}, \ldots, X_{r}\right)=\sum_{J \subseteq\{1,2, \ldots, r\},|J|=n} \prod_{j \in J} X_{j}
$$


be the $n$-th elementary symmetric polynomial, $n=0,1,2, \ldots, r$. Let

$$
\begin{aligned}
& \mathbf{H}_{n}\left(X_{1}, X_{2}, \ldots, X_{r}\right)=\sum_{\substack{t_{1}, t_{2}, \ldots, t_{r} \in \mathbb{Z}_{\geqslant 0} \\
t_{1}+t_{2}+\cdots+t_{r}=n}} X_{1}^{t_{1}} X_{2}^{t_{2}} \cdots X_{r}^{t_{r}} \quad \text { for all } n \in \mathbb{Z}, \\
& \mathbf{P}_{n}\left(X_{1}, X_{2}, \ldots, X_{r}\right)=\sum_{i=1}^{r} X_{i}^{n} \quad \text { for all } n \geqslant 1 .
\end{aligned}
$$

In particular, we have $\mathbf{E}_{0}=1, \mathbf{H}_{0}=1$, and $\mathbf{H}_{n}=0$ for $n<0$.

Proposition 3.9. Let $\xi_{1}, \xi_{2}, \ldots, \xi_{r}$ be the roots (not necessarily distinct) of the polynomial $F_{r}(X)$. Then we have

(1) $t_{r}(n)=\sum_{\substack{t_{1}, t_{2}, \ldots, t_{r} \in \mathbb{Z}_{\geqslant 0} \\ t_{1}+t_{2}+\cdots+t_{r}=n}} \xi_{1}^{t_{1}} \xi_{2}^{t_{2}} \cdots \xi_{r}^{t_{r}}$,

(2) $\widetilde{t}_{r}(n)=\sum_{i=1}^{r} \xi_{i}^{n}$.

Proof. Using Vieta's formula on symmetric polynomials, we have

$$
\mathbf{E}_{i}\left(\xi_{1}, \xi_{2}, \ldots, \xi_{r}\right)=(-1)^{i} c_{i}
$$

for $i=0,1,2, \ldots, r$. By [3, Lemma 4.8], we have

$$
\sum_{i=0}^{r} c_{i} \mathbf{H}_{n-i}\left(\xi_{1}, \xi_{2}, \ldots, \xi_{r}\right)=0
$$

On the other hand, Lemma 2.4 yields $t_{r}(n)=\sum_{i=1}^{r} C_{i-1} \cdot t_{r}(n-i)$ which implies

$$
\sum_{i=0}^{r} c_{i} \cdot t_{r}(n-i)=0
$$

Therefore

$$
\sum_{i=0}^{r} c_{i} \cdot\left(t_{r}(n-i)-\mathbf{H}_{n-i}\left(\xi_{1}, \xi_{2}, \ldots, \xi_{r}\right)\right)=0 .
$$

Note that $t_{r}(0)=1=\mathbf{H}_{0}\left(\xi_{1}, \xi_{2}, \ldots, \xi_{r}\right)$ and $t_{r}(n)=0=\mathbf{H}_{n}\left(\xi_{1}, \xi_{2}, \ldots, \xi_{r}\right)$ for $n<0$. Therefore, using induction and equation (3.1), we see that for all $n$

$$
t_{r}(n)=\mathbf{H}_{n}\left(\xi_{1}, \xi_{2}, \ldots, \xi_{r}\right)=\sum_{\substack{t_{1}, t_{2}, \ldots, t_{r} \in \mathbb{Z}_{\geqslant 0} \\ t_{1}+t_{2}+\cdots+t_{r}=n}} \xi_{1}^{t_{1}} \xi_{2}^{t_{2}} \cdots \xi_{r}^{t_{r}} .
$$

In Proposition 3.7, we have shown the following relation

$$
\tilde{t}_{r}(n)=\sum_{i=1}^{r}-i \cdot c_{i} \cdot t_{r}(n-i)
$$

Hence,

$$
\tilde{t}_{r}(n)=\sum_{i=1}^{r}-i \cdot c_{i} \cdot t_{r}(n-i)=\sum_{i=1}^{r}-i \cdot c_{i} \cdot \mathbf{H}_{n-i}\left(\xi_{1}, \xi_{2}, \ldots, \xi_{r}\right)
$$




$$
\begin{aligned}
& =\sum_{i=1}^{r}-i \cdot(-1)^{i} \cdot \mathbf{E}_{i}\left(\xi_{1}, \xi_{2}, \ldots, \xi_{r}\right) \cdot \mathbf{H}_{n-i}\left(\xi_{1}, \xi_{2}, \ldots, \xi_{r}\right) \\
& =\sum_{i=1}^{r}(-1)^{i-1} \cdot i \cdot \mathbf{E}_{i}\left(\xi_{1}, \xi_{2}, \ldots, \xi_{r}\right) \cdot \mathbf{H}_{n-i}\left(\xi_{1}, \xi_{2}, \ldots, \xi_{r}\right) \\
& =\mathbf{P}_{n}\left(\xi_{1}, \xi_{2}, \ldots, \xi_{r}\right) \quad(\text { by }[3, \text { Lemma } 4.8]) \\
& =\sum_{i=1}^{r} \xi_{i}^{n} .
\end{aligned}
$$

For $r=2$, we obtain the Fibonacci recurrence $\tilde{t}_{2}(n)=\tilde{t}_{2}(n-1)+\tilde{t}_{2}(n-2)$; however, with different initial conditions. Thus we obtain Lucas numbers and we have the following formula.

Corollary 3.10. $\tilde{t}_{2}(n)=\left(\frac{1+\sqrt{5}}{2}\right)^{n}+\left(\frac{1-\sqrt{5}}{2}\right)^{n}$.

\section{The number of support $\tau$-tilting modules over Nakayama algebras}

In this section, we will apply our results to give a new proof of a theorem by Asai, see [3, Theorem 4.1(1) and (2)]

Applying Proposition 3.2, we obtain the following recurrence relation for the number of support $\tau$-tilting modules over $\Lambda_{n}^{r}$. See Table 2 in Section 5 for explicit values of $s_{r}(n)$.

Proposition 4.1 ([3, Theorem 4.1(1)]).

$$
s_{r}(n)=2 s_{r}(n-1)+\sum_{i=2}^{r} C_{i-1} \cdot s_{r}(n-i) .
$$

Proof. Since $\Lambda=\Lambda_{n}^{r}$, we have $\Lambda_{<i} \cong \Lambda_{i-1}^{r}$ and $\Lambda_{>i} \cong \Lambda_{n-i}^{r}$. Thus Proposition 3.2(1) yields

$$
s_{r}(n)=\sum_{i=1}^{n} t_{r}(i-1) \cdot s_{r}(n-i)+t_{r}(n) .
$$

Therefore,

$$
\begin{aligned}
s_{r}(n)-s_{r}(n-1)= & \sum_{i=1}^{n} t_{r}(i-1) \cdot s_{r}(n-i)+t_{r}(n) \\
& -\left(\sum_{i=1}^{n-1} t_{r}(i-1) \cdot s_{r}(n-1-i)+t_{r}(n-1)\right) \\
= & s_{r}(n-1)+\sum_{i=1}^{n-1}\left(t_{r}(i)-t_{r}(i-1)\right) \cdot s_{r}(n-1-i)+\left(t_{r}(n)-t_{r}(n-1)\right) .
\end{aligned}
$$

By Lemma 2.4, for all $i$ we have

$$
t_{r}(i)=t_{r}(i-1)+\sum_{\ell=2}^{r} C_{\ell-1} \cdot t_{r}(i-\ell) .
$$

Using this equality in the identity above, we get

$$
s_{r}(n)-s_{r}(n-1)=s_{r}(n-1)+\sum_{i=1}^{n-1} \sum_{\ell=2}^{r} C_{\ell-1} \cdot t_{r}(i-\ell) \cdot s_{r}(n-1-i)
$$




$$
+\sum_{\ell=2}^{r} C_{\ell-1} \cdot t_{r}(n-\ell)
$$

and since $s_{r}(n-\ell-i)=0$ whenever $i>n-\ell$, we obtain

$$
\begin{aligned}
s_{r}(n)-s_{r}(n-1)= & s_{r}(n-1)+\sum_{\ell=2}^{r} C_{\ell-1} \cdot \sum_{i=1}^{n-\ell} t_{r}(i-1) \cdot s_{r}(n-\ell-i) \\
& +\sum_{\ell=2}^{r} C_{\ell-1} \cdot t_{r}(n-\ell) \\
= & s_{r}(n-1)+\sum_{\ell=2}^{r} C_{\ell-1} \cdot\left(\sum_{i=1}^{n-\ell} t_{r}(i-1) \cdot s_{r}(n-\ell-i)+t_{r}(n-\ell)\right) \\
= & s_{r}(n-1)+\sum_{\ell=2}^{r} C_{\ell-1} \cdot s_{r}(n-\ell) \quad \text { by Proposition 3.2(1). }
\end{aligned}
$$

Hence, $s_{r}(n)=2 s_{r}(n-1)+\sum_{i=2}^{r} C_{i-1} \cdot s_{r}(n-i)$.

Remark 4.2. If $r \geq n$ then $\Lambda_{n}^{r}$ is a hereditary algebra of Dynkin type $\mathbb{A}_{n}$ and the support $\tau$-tilting modules are in bijection with the clusters in the corresponding cluster algebra. In particular, $s_{r}(n)=C_{n+1}$. This fact also can be obtained directly from Proposition 4.1 and the identity $C_{n}=\sum_{i=1}^{n} C_{i-1} \cdot C_{n-i}$ (see Remark 2.5). Indeed, the following equation

$$
\begin{aligned}
s_{n}(n)-C_{n+1}= & 2 s_{n}(n-1)+\sum_{i=2}^{n} C_{i-1} \cdot s_{n}(n-i)-\left(\sum_{i=1}^{n+1} C_{i-1} \cdot C_{n+1-i}\right) \\
= & C_{0} \cdot s_{n}(n-1)+\sum_{i=2}^{n} C_{i-1} \cdot s_{n}(n-i)-\left(\sum_{i=1}^{n} C_{i-1} \cdot C_{n+1-i}\right) \\
& +s_{n}(n-1)-C_{n} \cdot C_{0} \\
= & \sum_{i=1}^{n} C_{i-1} \cdot\left(s_{n}(n-i)-C_{n+1-i}\right)+\left(s_{n}(n-1)-C_{n} \cdot C_{0}\right) \\
= & \sum_{i=1}^{n} C_{i-1} \cdot\left(s_{n-i}(n-i)-C_{n+1-i}\right)+\left(s_{n-1}(n-1)-C_{n}\right)
\end{aligned}
$$

implies $s_{n}(n)=C_{n+1}$ by induction.

Applying Theorem 3.8, we obtain the following recurrence relation for the number of support $\tau$-tilting modules over $\widetilde{\Lambda}_{n}^{r}$. See Table 4 in Section 5 for explicit values of $\widetilde{s}_{r}(n)$.

Proposition 4.3 ([3, Theorem 4.1(2)]).

$$
\tilde{s}_{r}(n)=2 \tilde{s}_{r}(n-1)+\sum_{i=2}^{r} C_{i-1} \cdot \tilde{s}_{r}(n-i) .
$$

Proof. We need to show

$$
\tilde{s}_{r}(n)-2 \tilde{s}_{r}(n-1)-\sum_{i=2}^{r} C_{i-1} \cdot \tilde{s}_{r}(n-i)=0 .
$$


Using Proposition 3.5 and the equation $\tilde{s}_{r}(n)=\mid \operatorname{ps} \tau$-tilt $\widetilde{\Lambda}_{n}^{r} \mid+\tilde{t}_{r}(n)$, we obtain

$$
\tilde{s}_{r}(n)=\sum_{\ell=1}^{n-1} \ell \cdot t_{r}(\ell-1) \cdot s_{r}(n-\ell-1)+n \cdot t_{r}(n-1)+\tilde{t}_{r}(n) .
$$

Therefore,

$$
\begin{aligned}
& \tilde{s}_{r}(n)-2 \tilde{s}_{r}(n-1)-\sum_{i=2}^{r} C_{i-1} \cdot \tilde{s}_{r}(n-i) \\
& =\sum_{\ell=1}^{n-1} \ell \cdot t_{r}(\ell-1) \cdot s_{r}(n-\ell-1)+n \cdot t_{r}(n-1)+\tilde{t}_{r}(n) \\
& \quad-2\left(\sum_{\ell=1}^{n-2} \ell \cdot t_{r}(\ell-1) \cdot s_{r}(n-\ell-2)+(n-1) \cdot t_{r}(n-2)+\tilde{t}_{r}(n-1)\right) \\
& \quad-\sum_{i=2}^{r} C_{i-1} \cdot\left(\sum_{\ell=1}^{n-i-1} \ell \cdot t_{r}(\ell-1) \cdot s_{r}(n-i-\ell-1)+(n-i) \cdot t_{r}(n-i-1)+\tilde{t}_{r}(n-i)\right) .
\end{aligned}
$$

Note that every term in the summation $\sum_{i=2}^{r} C_{i-1} \sum_{\ell=n-i}^{n-2} \ell \cdot t_{r}(\ell-1) \cdot s_{r}(n-i-\ell-1)$ is zero, and therefore the whole expression is equal to

$$
\begin{aligned}
= & \sum_{\ell=1}^{n-2} \ell \cdot t_{r}(\ell-1) \cdot\left(s_{r}(n-\ell-1)-2 s_{r}(n-\ell-2)-\sum_{i=2}^{r} C_{r-1} \cdot s_{r}(n-\ell-1-i)\right) \\
& +n \cdot t_{r}(n-1)+\left((n-1) \cdot t_{r}(n-2)-2(n-1) \cdot t_{r}(n-2)\right) \\
& -\sum_{i=2}^{r}(n-i) \cdot C_{i-1} \cdot t_{r}(n-i-1)+\left(\tilde{t}_{r}(n)-2 \tilde{t}_{r}(n-1)-\sum_{i=2}^{r} C_{i-1} \cdot \tilde{t}_{r}(n-i)\right) .
\end{aligned}
$$

Now, the parenthesis in the first sum is zero, by Proposition 4.1, the large parenthesis in the second row can be included in the first sum of the third row as the $i=1$ term, and the parenthesis in the third row is equal to $-\tilde{t}_{r}(n-1)$ by Theorem 3.8. So the whole expression is equal to

$$
\begin{aligned}
& =n \cdot t_{r}(n-1)-\sum_{i=1}^{r}(n-i) \cdot C_{i-1} \cdot t_{r}(n-i-1)-\tilde{t}_{r}(n-1) \\
& =n \cdot\left(t_{r}(n-1)-\sum_{i=1}^{r} C_{i-1} \cdot t_{r}(n-i-1)\right)+\sum_{i=1}^{r} i \cdot C_{i-1} \cdot t_{r}(n-i-1)-\tilde{t}_{r}(n-1) \\
& =\sum_{i=1}^{r} i \cdot C_{i-1} \cdot t_{r}(n-i-1)-\tilde{t}_{r}(n-1) \quad(\text { by Lemma 2.4) } \\
& =\tilde{t}_{r}(n-1)-\tilde{t}_{r}(n-1) \quad(\text { by Proposition } 3.7) \\
& =0 .
\end{aligned}
$$

\section{$5 \quad$ Examples}

In this section, we give examples of the numbers of (support) $\tau$-tilting modules over $\Lambda_{n}^{r}$ and $\widetilde{\Lambda}_{n}^{r}$ (see Tables 1-4). 
Table 1. The number of $\tau$-tilting modules of $\Lambda_{n}^{r}$.

\begin{tabular}{|c|c|c|c|c|c|c|c|c|c|c|c|c|}
\hline$t_{r}(n) \backslash n$ & 1 & 2 & 3 & 4 & 5 & 6 & 7 & 8 & 9 & 10 & 11 & 12 \\
\hline 1 & 1 & 1 & 1 & 1 & 1 & 1 & 1 & 1 & 1 & 1 & 1 & 1 \\
\hline 2 & 1 & 2 & 3 & 5 & 8 & 13 & 21 & 34 & 55 & 89 & 144 & 233 \\
\hline 3 & 1 & 2 & 5 & 9 & 18 & 37 & 73 & 146 & 293 & 585 & 1170 & 2341 \\
\hline 4 & 1 & 2 & 5 & 14 & 28 & 62 & 143 & 331 & 738 & 1665 & 3780 & 8576 \\
\hline 5 & 1 & 2 & 5 & 14 & 42 & 90 & 213 & 527 & 1326 & 3317 & 8022 & 19608 \\
\hline 6 & 1 & 2 & 5 & 14 & 42 & 132 & 297 & 737 & 1914 & 5081 & 13566 & 35862 \\
\hline
\end{tabular}

Table 2. The number of support $\tau$-tilting modules of $\Lambda_{n}^{r}$.

\begin{tabular}{|c|c|c|c|c|c|c|c|c|c|c|c|c|}
\hline$s_{r}(n) \backslash n$ & 1 & 2 & 3 & 4 & 5 & 6 & 7 & 8 & 9 & 10 & 11 & 12 \\
\hline 1 & 2 & 4 & 8 & 16 & 32 & 64 & 128 & 256 & 512 & 1024 & 2048 & 4096 \\
\hline 2 & 2 & 5 & 12 & 29 & 70 & 169 & 408 & 985 & 2378 & 5741 & 13860 & 33461 \\
\hline 3 & 2 & 5 & 14 & 37 & 98 & 261 & 694 & 1845 & 4906 & 13045 & 34686 & 92229 \\
\hline 4 & 2 & 5 & 14 & 42 & 118 & 331 & 934 & 2645 & 7476 & 21120 & 59676 & 168649 \\
\hline 5 & 2 & 5 & 14 & 42 & 132 & 387 & 1130 & 3317 & 9786 & 28932 & 85352 & 251613 \\
\hline 6 & 2 & 5 & 14 & 42 & 132 & 429 & 1298 & 3905 & 11802 & 35862 & 109376 & 333933 \\
\hline
\end{tabular}

Table 3. The number of $\tau$-tilting modules of $\widetilde{\Lambda}_{n}^{r}$.

\begin{tabular}{|c|c|c|c|c|c|c|c|c|c|c|c|c|}
\hline$\tilde{t}_{r}(n) \backslash n$ & 1 & 2 & 3 & 4 & 5 & 6 & 7 & 8 & 9 & 10 & 11 & 12 \\
\hline 1 & 1 & 1 & 1 & 1 & 1 & 1 & 1 & 1 & 1 & 1 & 1 & 1 \\
\hline 2 & 1 & 3 & 4 & 7 & 11 & 18 & 29 & 47 & 76 & 123 & 199 & 322 \\
\hline 3 & 1 & 3 & 10 & 15 & 31 & 66 & 127 & 255 & 514 & 1023 & 2047 & 4098 \\
\hline 4 & 1 & 3 & 10 & 35 & 56 & 126 & 302 & 715 & 1549 & 3498 & 7897 & 18158 \\
\hline 5 & 1 & 3 & 10 & 35 & 126 & 210 & 498 & 1275 & 3313 & 8398 & 19691 & 48062 \\
\hline 6 & 1 & 3 & 10 & 35 & 126 & 462 & 792 & 1947 & 5203 & 14278 & 39095 & 104006 \\
\hline
\end{tabular}

Table 4. The number of support $\tau$-tilting modules of $\widetilde{\Lambda}_{n}^{r}$.

\begin{tabular}{|c|c|c|c|c|c|c|c|c|c|c|c|c|}
\hline$\tilde{s}_{r}(n) \backslash n$ & 1 & 2 & 3 & 4 & 5 & 6 & 7 & 8 & 9 & 10 & 11 & 12 \\
\hline 1 & 2 & 4 & 8 & 16 & 32 & 64 & 128 & 256 & 512 & 1024 & 2048 & 4096 \\
\hline 2 & 2 & 6 & 14 & 34 & 82 & 198 & 478 & 1154 & 2786 & 6726 & 16238 & 39202 \\
\hline 3 & 2 & 6 & 20 & 50 & 132 & 354 & 940 & 2498 & 6644 & 17666 & 46972 & 124898 \\
\hline 4 & 2 & 6 & 20 & 70 & 182 & 504 & 1430 & 4078 & 11504 & 32466 & 91742 & 259348 \\
\hline 5 & 2 & 6 & 20 & 70 & 252 & 672 & 1920 & 5646 & 16796 & 49966 & 147028 & 432724 \\
\hline 6 & 2 & 6 & 20 & 70 & 252 & 924 & 2508 & 7326 & 22088 & 67606 & 208012 & 638356 \\
\hline
\end{tabular}

\section{Acknowledgements}

The first author was partially supported by NSFC (Grant No. 11971225). The second author was supported by the NSF grant DMS-1800860 and by the University of Connecticut. The authors also thank the referees for the useful and detailed suggestions. 


\section{References}

[1] Adachi T., The classification of $\tau$-tilting modules over Nakayama algebras, J. Algebra 452 (2016), 227-262, arXiv:1309.2216.

[2] Adachi T., Iyama O., Reiten I., $\tau$-tilting theory, Compos. Math. 150 (2014), 415-452, arXiv:1210.1036.

[3] Asai S., Semibricks, Int. Math. Res. Not., to appear, arXiv:1610.05860.

[4] Assem I., Brüstle T., Schiffler R., Cluster-tilted algebras as trivial extensions, Bull. Lond. Math. Soc. 40 (2008), 151-162, arXiv:math.RT/0601537.

[5] Assem I., Simson D., Skowroński A., Elements of the representation theory of associative algebras, Vol. 1, Techniques of representation theory, London Mathematical Society Student Texts, Vol. 65, Cambridge University Press, Cambridge, 2006.

[6] Auslander M., Smalø S.O., Almost split sequences in subcategories, J. Algebra 69 (1981), 426-454.

[7] Buan A.B., Marsh R., Reineke M., Reiten I., Todorov G., Tilting theory and cluster combinatorics, Adv. Math. 204 (2006), 572-618, arXiv:math.RT/0402054.

[8] Caldero P., Chapoton F., Schiffler R., Quivers with relations arising from clusters ( $A_{n}$ case), Trans. Amer. Math. Soc. 358 (2006), 1347-1364, arXiv:math.RT/0401316.

[9] Fomin S., Zelevinsky A., Y-systems and generalized associahedra, Ann. of Math. 158 (2003), 977-1018, arXiv:hep-th/0111053.

[10] Happel D., Ringel C.M., Tilted algebras, Trans. Amer. Math. Soc. 274 (1982), 399-443.

[11] Keller B., Vossieck D., Aisles in derived categories, Bull. Soc. Math. Belg. Sér. A 40 (1988), $239-253$.

[12] Obaid M.A.A., Nauman S.K., Fakieh W.M., Ringel C.M., The number of support-tilting modules for a Dynkin algebra, J. Integer Seq. 18 (2015), Article 15.10.6, 24 pages, arXiv:1403.5827.

[13] Schiffler R., Quiver representations, CMS Books in Mathematics, Springer, Cham, 2014. 